Internist 2021 - 62:461-462

https://doi.org/10.1007/s00108-021-01026-9

Angenommen: 23. März 2021

๑) Springer Medizin Verlag GmbH, ein Teil von Springer Nature 2021

Die muskuloskeletale Integrität des Bewegungsapparats ist einer der Faktoren, die für uns lebensbestimmend sind. Durch Aufrechterhaltung der Mobilität, Geschicklichkeit und Fähigkeit zu aktiver Lebensgestaltung determiniert sie die physische und psychische Gesundheit und Autonomie des Subjekts. Darüber hinaus beeinflusst die muskuloskeletale Gesundheit in relevantem Umfang Langzeitmorbidität und Mortalität.

》) Die COVID-19-Pandemie hat gravierende Auswirkungen auf die Betreuung von Patienyten mit Osteoporose

Osteoporose gehört zu den Volkskrankheiten. Laut der schon etwas älteren European Prospective Osteoporosis Study (EPOS) liegt in Deutschland bei 15\% postmenopausaler Frauen im Alter von 50 bis 60 Jahren und bei $45 \%$ der über 70 Jährigen eine erniedrigte Knochendichte vor (Definition der Weltgesundheitsorganisation für Osteoporose mittels Dualröntgenabsorptiometrie[DXA]-T-

Score $\leq-2,5)$. Auch Männer sind häufig betroffen: Eine entsprechend erniedrigte Knochendichte am Schenkelhals findet sich bei $2,4 \%$ der 50 - bis 60 Jährigen und bei $17 \%$ der über 70 Jährigen [1]. Zusammengenommen besteht damit, auch auf der Grundlage von Versicherungsdaten, bei etwa 6,3 Mio. Bundesbürgern aktuell eine Osteoporose, definiert durch niedrige Knochendichte, osteoporotische Fraktur oder eine Osteoporosemedikation [2].

Hiermit einher geht eine Zunahme der Osteosarkopenie, unter der wir das gleichzeitige Vorliegen von Osteoporo-

\author{
Martin Reincke ${ }^{1} \cdot$ Hendrik Lehnert ${ }^{2}$ \\ ${ }^{1}$ Medizinische Klinik und Poliklinik IV, Klinikum der Universität, Ludwig-Maximilians-Universität München, \\ München, Deutschland \\ ${ }^{2}$ Paris Lodron Universität Salzburg, Salzburg, Österreich
}

\title{
Osteologie im Blickpunkt
}

se und Sarkopenie verstehen. Letztere ist dabei definiert als Verlust von Muskelmasse und Funktion. Pathophysiologisch immer noch unzureichend verstanden, beträgt die Prävalenz bei älteren Erwachsenen $>65$ Jahre 5-37\%, und bei höherem Lebensalter plus Osteoporosefrakturen sogar bis $46 \%$ [3].

Aktuelle Umfragen bei Patienten und Beschäftigten in unterschiedlichen Gesundheitssystemen weltweit zeigen übereinstimmend, dass die COVID-19Pandemie gravierende Auswirkungen auf die Betreuung von Patienten mit chronischen Erkrankungen hat. Dies trifft besonders auch auf Patienten mit Osteoporose zu, da sie altersbedingt zur besonders vulnerablen Risikopopulation gehören. Fragebogengestützte Erhebungen in den USA und weltweit zeigen, dass es in der Pandemie zu einem drastischen Rückgang der Osteoporosekonsultationen um bis zu 70 \% kam, ohne dass telefonische Konsultationen und Videokonsultationen diese Ausfälle hätten kompensieren können. Neben drastisch rückläufigen DXA-Messungen wurde auch über Schwierigkeiten bei der Medikamentenversorgung bzw. der parenteralen Medikamentenapplikation berichtet $[4,5]$.

Dies sind alles gute Gründe, dass sich die vorliegende Ausgabe thematisch mit osteologischen Erkrankungen beschäftigt. Der erste Beitrag von Thomasius u. Bühring beschäftigt sich mit der Prädiktion des Frakturrisikos. Diverse Risikoscores wurden in den letzten Jahren entwickelt und validiert. Hiermit ist es jetzt möglich, für definierte $\mathrm{Ri}$ sikopopulationen das Frakturrisiko zu bestimmen. Für Deutschland hat sich das Modell des Dachverbands Osteologie etabliert, mit dem sich das 10Jahres-Risiko von Wirbelkörper- oder
Hüftfrakturen abschätzen lässt. Entsprechend dem Leitlinienalgorithmus entfällt bei einem 10-Jahres-Risiko $\leq 20 \%$ eine weitere Diagnostik, bei $>20 \%$ besteht eine Indikation zur Basisdiagnostik und bei $>30 \%$ sind Basisdiagnostik und spezifische Osteoporosetherapie indiziert.

Der Beitrag von Obermayer-Pietsch, Fössl u. Dimai behandelt wichtige Fragen der Osteoporosetherapie, die sich aus der inzwischen sehr umfangreichen Literatur ergeben. So bestehen heute klare Empfehlungen zur Dauer der jeweiligen Therapie und zum Vorgehen bei Pausierung bzw. Beendigung der Therapie. Ebenfalls seit ein paar Jahren in den Fokus gerückt sind die Sequenz- und Kombinationstherapie, mit denen die Wirksamkeit der Osteoporosetherapien erhöht werden soll, optimalerweise bei Reduktion der substanzspezifischen Nebenwirkungen.

Die Übersichtsarbeit von Seefried u. Jakob stellt die neuesten Erkenntnisse zu seltenen osteologischen Erkrankungen dar. Die molekulare Pathophysiologie von Hypophosphatasie, X-chromosomaler Hypophosphatämie und Osteogenesis imperfecta ist seit Längerem aufgeklärt, und wirksame Therapien für die monogenetischen Krankheitsbilder, insbesondere bei Vorliegen eines schweren Phänotyps, sind etabliert.

\section{》) Operative Behandlungs- bedürftigkeit bei primärem Hyperparathyreoidismus wird kontrovers diskutiert}

Der primäre Hyperparathyreoidismus ist heute in mehr als $90 \%$ der Fälle asymptomatisch und wird zufällig im Rahmen von Laborchecks diagnostiziert, wie der 
Beitrag von Gollisch u. Siggelkow ausführt. Die Frage der operativen Behandlungsbedürftigkeit beschäftigt die Community schon seit 20 Jahren, wobei sich leider ein gewisser Dissens zwischen den chirurgischen und endokrinologischen Kollegen in den letzten Jahren eher vertieft hat. Dies ist allerdings auch der immer noch unzureichenden Datenlage geschuldet, die der Willkür von Empfehlungen Tür und Tor öffnet. Es ist $\mathrm{zu}$ hoffen, dass mit der augenblicklich in Angriff genommenen S2k-Leitlinie der Deutschen Gesellschaft für Endokrinologie zum Wohl der Patienten ein interdisziplinärer Konsensus wiederhergestellt werden wird.

Besonderheiten geriatrischer Patienten bei osteologischen Fragestellungen sind mit erheblicher Verspätung in den Fokus der klinischen Praxis gerückt. So ist es inzwischen Common Sense, dass die üblichen Diagnoseverfahren einer Osteoporose bei hochbetagten und gebrechlichen Personen versagen können. Zudem spielt pathophysiologisch die Sarkopenie eine tragende Rolle bei Frakturursache und Frakturheilung. Die „unhappy triad“ - Gebrechlichkeit, Alter und Osteoporose - führt zu Stürzen, Frakturen, Behinderung und Mortalität, wie die Übersichtsarbeit von Drey u. Schmidmaier ausführt. Die Kombination der Knochendichtemessung mit einer Analyse der Gesamtkörperzusammensetzung mittels DXA erfasst nicht nur die kritische Minderung des Knochenmineralgehalts, sondern gleichzeitig auch quantitativ und reliabel die Abnahme der Muskulatur.

Wir hoffen, dass es uns gelungen ist, für Sie als Leserinnen und Leser der Zeitschrift Der Internist eine informative und praxisrelevante Ausgabe zusammenzustellen. Viel Freude beim Lesen!,

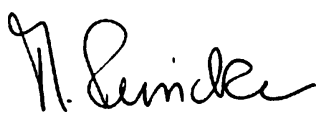

M. Reincke,<smiles>CCCCCCC</smiles>

H. Lehnert

\section{Korrespondenzadresse}

Prof. Dr. Martin Reincke

Medizinische Klinik und Poliklinik IV, Klinikum der Universität, Ludwig-MaximiliansUniversität München

Ziemssenstr. 1, 80336 München, Deutschland martin.reincke@med.uni-muenchen.de

\section{Prof. Dr. Hendrik Lehnert}

Paris Lodron Universität Salzburg

Kapitelgasse 4/I, 5020 Salzburg, Österreich

hendrik.lehnert@sbg.ac.at

Interessenkonflikt. M. Reincke und H. Lehnert geben an, dass kein Interessenkonflikt besteht.

\section{Literatur}

1. Scheidt-Nave C, Banzer D (1997) Abendroth K Schlussbericht Multizentrische Studie zu Verteilung, Determination und prädiktivem Wert der Knochendichte in der deutschen Bevölkerung Förderprojekt des Bundesministeriums für Forschung und TechnologieFörderkennzeichen 01KM 9304/0, S1-45

2. Hadji P1, Klein S, Gothe H, Häussler B, Kless T, Schmidt T, Steinle T, Verheyen F, Linder R. The epidemiology of osteoporosis-Bone Evaluation Study (BEST): an analysis of routine health insurance data. Dtsch Arztebl Int. 2013 Jan; 110(4):52-7. https://doi.org/10.3238/arztebl. 2013.0052. Epub 2013 Jan 25.

3. Kirk B, Zanker J, Osteosarcopenia DG (2020) epidemiology, diagnosis, and treatment-facts and numbers. J Cachexia Sarcopenia Muscle 11(3):609-618. https://doi.org/10.1002/jcsm. 12567 (Epub 2020 Mar 22. PMID: 32202056 Free PMC article)

4. Singer AJ, Fuggle NR, Gill CB, Patel AR, Medeiros AP, Greenspan SL. COVID-19 and effects on osteoporosis management: the patient perspective from a National Osteoporosis Foundation survey. Osteoporos Int. 2021 Feb 8:1-4. https://doi.org/10. 1007/s00198-021-05836-3. Online im Druck.

5. Fuggle NR, Singer A, Gill C, Patel A, Medeiros A, Mlotek AS, Pierroz DD, Halbout P, Harvey CN, Reginster JY, Cooper C, Greenspan SL (2021) How has COVID-19 affected the treatment of osteoporosis? An IOF-NOF-ESCEO global survey. Osteoporos Int 8:1-7. https://doi.org/10.1007/ s00198-020-05793-3

\section{Risikofaktoren für schwere COVID-19-Verläufe bei Rheumapatienten}

Erste Daten aus dem COVID-19-Register der DGRh definieren Risikofaktoren für schwere Verläufe, die mit einer rheumatischen Grunderkrankung zusammenhängen.

Sind Rheumapatienten bei einer SARSCoV-2-Infektion besonderen Risiken ausgesetzt und welchen Einfluss hat die Rheumamedikation? Um diese Fragen zu klären, hat die Deutsche Gesellschaft für Rheumatologie e. V. (DGRh) gemeinsam mit der Universität Gießen das Online-Register "Covid19-rheuma.de" ins Leben gerufen. In diesem werden Covid-19-Krankheitsverläufe von Patienten mit Rheuma dokumentiert.

Dabei bestätigen sich zunächst die auch für die Allgemeinbevölkerung geltenden Erkenntnisse zu SARS-CoV-2: Als unabhängiger Risikofaktor für einen schweren Verlauf zeigte sich besonders das Alter über 65-Jährige hatten ein 2,24-mal, über 75-Jährige sogar ein fast 4-mal so hohes Hospitalisierungsrisiko wie jüngere Patienten. Auch die Art und Anzahl der zusätzlichen Begleiterkrankungen beeinflusste den Verlauf der Erkrankung deutlich.

Daneben lassen sich aber auch Risikofaktoren ausmachen, die speziell mit einer rheumatischen Grunderkrankung und ihrer Therapie in Verbindung stehen: Als Risikogruppe erwiesen sich insbesondere Patienten, die täglich mehr als 5 mg Glukokortikoide einnahmen, sowie solche, deren aktuelle Krankheitsaktivität als moderat bis hoch eingeschätzt wurde.

Während der Pandemie sollte daher auf eine möglichst gute medikamentöse Kontrolle der rheumatischen Grunderkrankung geachtet werden und, wo immer möglich, auf die dauerhafte Gabe höher dosierter Glukokortikoide verzichtet werden.

Quelle: Deutsche Gesellschaft für Rheumatologie e. V., www.dgrh.de 